\title{
Diseño de un sistema de producción modular en una mediana empresa de confecciones
}

\section{INTRODUCCIÓN}

Las exigencias del mercado actual obligan a las empresas a buscar permanentemente nuevos enfoques y métodos de producción que permitan flexibilizar los procesos para responder de manera rápida a los clientes, con niveles adecuados de calidad, servicio y precio. En este caso, el tipo de producción utilizado en la confección de prendas influye sobre los indicadores de desempeño aquí mencionados.

La producción modular es un sistema de producción flexible basado en la filosofía "justo a tiempo", que pretende desarrollar una producción halada por el cliente y administrada por un equipo de trabajadores polivalentes que aplican la mejora continua en los procesos a su cargo. Los principales beneficios obtenidos por dicha producción son: reducción del tiempo de reposición de un lote, reducción del inventario en proceso, mejoramiento de la calidad, mejora del servicio y optimización del uso de espacios. El tamaño de lote ideal de este sistema de producción es "1", lo que le permite flexibilidad frente a los cambios del mercado. Los centros de producción modular suelen conformarse por grupos de 6 a 18 trabajadores compatibles, de actitud positiva, capacitados para ejecutar tres o más operaciones diferentes con calidad y eficiencia. 
La empresa textil de confecciones, a diferencia de otras, debe someterse a la dinámica variación de la moda y a la difícil competencia de novedosos productos que con un bajo precio son ofrecidos en el mercado global. Esta situación proporciona una gran oportunidad de mejora a los sistemas de producción, y, para ello, una de las respuestas efectivas es el sistema de producción modular.

El sector textil de confecciones del Perú representa el 7\% del PBI de la industria manufacturera y su inversión por puesto de trabajo es de aproximadamente US\$1.500. Este sector es intensivo en el uso de mano de obra, pues ocupa - de manera directa e indirecta- al 10\% de la población del Perú y representa un porcentaje significativo de las exportaciones; por ejemplo, en el 2000 alcanzaron el 7,5\% de las exportaciones totales. Sin embargo, el 95\% de las empresas dedicadas a la confección de prendas mantienen el tradicional sistema de producción por lotes (bultos), lo cual les impide lograr un adecuado nivel de competitividad.

Así, la investigación que da motivo a este artículo se propuso los siguientes objetivos:

- Presentar un diseño modular para una mediana empresa textil de confecciones del Perú.

- Medir los beneficios técnicos derivados de la implantación del sistema de producción modular, haciendo uso de técnicas de simulación.

La hipótesis principal de la investigación plantea que la producción modular con transferencia mano a mano es una alternativa viable y beneficiosa para el sector textil de confecciones, que permitirá flexibilizar la producción y mejorar los principales indicadores técnicos de desempeño.

\section{MARCO CONCEPTUAL}

La producción flexible es el concepto primario que antecede al sistema de producción modular, concepto que se introduce formalmente en la década de 1970 en el Japón, donde la crisis del petróleo de 1973 colocó a las empresas de este país en una situación deficitaria, indicándose un único camino posible: la reducción de los costos. Taichi Ohno, vicepresidente de la empresa Toyota, creó una filosofía integral de producción, el Sistema de Producción Toyota (TPS), cuyo objetivo principal era la reducción de los costos superfluos debidos al despilfarro de ma- 
teriales, mano de obra y máquinas. Como objetivos secundarios se planteó el aprovechamiento de las capacidades humanas, la mejora de la calidad y la satisfacción de las necesidades de los clientes. La denominación genérica del enfoque Toyota fue la de "justo a tiempo" (JIT), dicha filosofía puede resumirse como "producir la cantidad requerida en el momento preciso, con la calidad adecuada, para satisfacer las necesidades del cliente, al menor costo posible".

En la década de 1980 el enfoque Toyota se empezó a aplicar en un gran número de empresas japonesas, americanas y europeas; es así como en el sector manufacturero, específicamente en el sector textil de la confección, aparece el concepto de "producción modular". Aplicaciones en este sentido han agrupado una variedad de configuraciones y características de sistemas bajo el lema de producción modular. Sobre la base de las características del sistema en el que el trabajo pasa directamente de mano en mano, y en algunos casos en los que las operarias trabajan de pie (Sistema de Costura Toyota), la producción modular es un tipo especial de producción que debiera diferenciarse de otros tipos radicalmente diferentes, como es el caso de la "costura en equipo". Un concepto básico que se utiliza en las decisiones de la producción modular, pasando de mano en mano, es el de balanceo de líneas de producción.

\section{Gráfico 1}

Ventajas del sistema modular con trabajo mano a mano, frente al sistema tradicional de confección de bulto progresivo (por lotes)

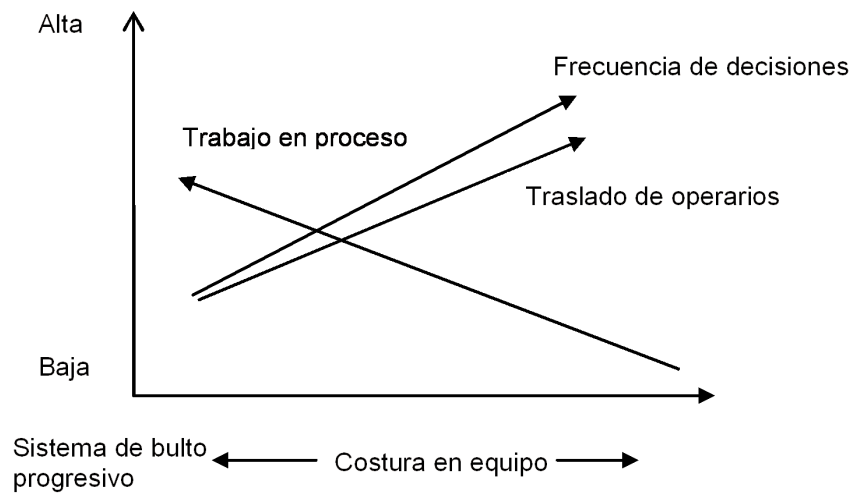


El gráfico 1 muestra que la continuidad de la producción modular reduce el inventario de prendas en proceso pero incrementa la movilización del trabajador, que se traslada con la prenda de una estación a otra. Toma una prenda cuando la anterior queda concluida.

La modularización pretende una producción esbelta (ligera) administrada por personal polivalente. Un módulo de procesamiento integra equipos de trabajadores, que desarrollarán todas las operaciones necesarias para la obtención de la prenda, que forma parte de un flujo continuo y flexible porque puede combinar modelos pertenecientes a una misma familia. Una decisión primordial es definir los procesos que se integrarán en los módulos, para posteriormente disponer las estaciones sobre la base de un balance de producción previamente calculado.

En la industria de la confección este proceso no demanda una considerable inversión de dinero ni de tiempo, pues su implementación puede ser gradual y los principales rubros de inversión estarían justificados por los requerimientos de capacitación y sensibilización del personal y el proceso de redistribución de recursos. El retorno de la inversión estará ampliamente cubierto por el incremento de la productividad, reducción significativa de los inventarios en proceso y reducción de infraestructura (máquina o equipo).

Existen políticas definidas para el diseño modular que serán explicadas con el diagrama de operaciones para una prenda "P":

- Cada operación requiere de una máquina.

- La prenda debe ser completada al pasar por la secuencia 1, 2, 3, 4.

- Un operario trabaja en una determinada máquina en un momento dado.

- El operario no debe ser interrumpido hasta terminar una determinada prenda.

- Todos los operarios trabajan con una eficiencia del 100\%.

- Las piezas de las prendas cortadas siempre están frente a la estación de operación 1. 


\section{DOP: Elaboración de prenda P}

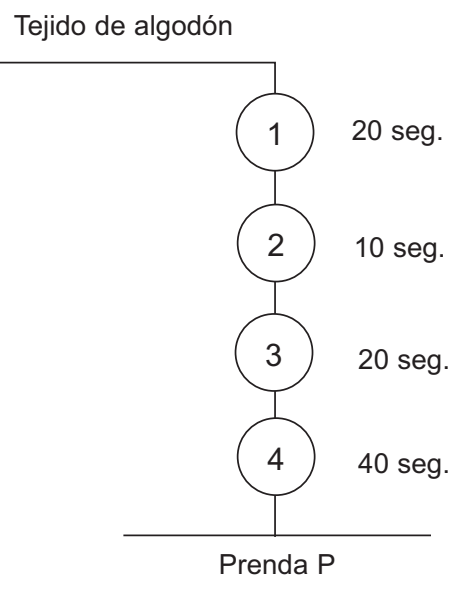

Para la secuencia mostrada tenemos las siguientes alternativas de agrupación modular:

1. Módulo con un solo operario

2. Módulo con dos operarios

3. Módulo con tres operarios

4. Módulo con cuatro operarios

\section{Gráfico 2}

Comparación de las cuatro alternativas de agrupación modular

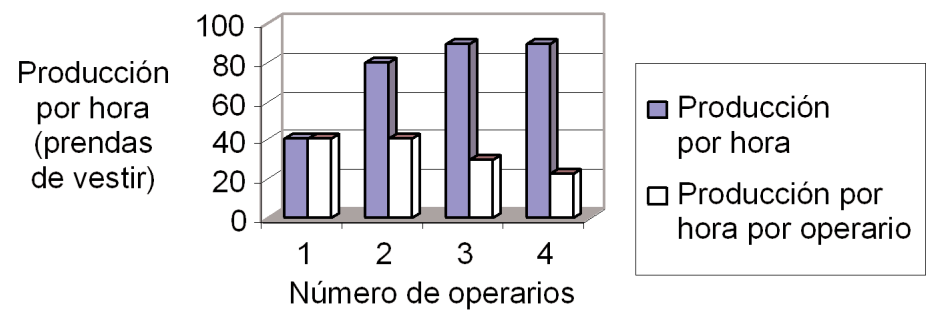

Como puede observarse la producción total por hora se incrementa en forma proporcional a la cantidad de operarios adicionados al módulo.

La implantación de módulos de producción requiere de las siguientes etapas: 
a. Sensibilización y compromiso del personal.

b. Capacitación y entrenamiento del personal.

c. Programación, tamaño de lote y secuenciación de la producción.

d. Establecimiento del módulo piloto y simulación.

e. Preparación de las especificaciones de calidad.

f. Puesta en marcha.

g. Ampliación del sistema a toda la planta.

\section{METODOLOGÍA Y MATERIALES}

\subsection{Tipo de investigación}

Se trata de una investigación aplicada en el área de producción de una mediana empresa de la confección dedicada a la producción de polos (T-Shirts), con una demanda mensual de prendas de 20.944 unidades. El método utilizado es el de análisis y síntesis. Las técnicas principales que se utilizarán son la observación, el muestreo y las encuestas.

\subsection{Muestra}

El sistema de producción actual fue observado durante una muestra de tiempo de tres meses. La información escrita incluyó órdenes de producción, hojas de ruta, guías de ingreso de productos terminados y reportes de control de calidad. Las observaciones iniciales consistieron en describir y medir el proceso de producción actual, al que se le tipifica como un sistema de producción por bultos o por lotes; así mismo, se cuantificaron los indicadores operacionales importantes, derivados de la administración vigente: productividad de la mano de obra, lead time de producción, nivel de calidad, nivel de servicio, trabajo en proceso (WIP), utilización de máquinas y equipos, clima laboral y compromiso con la calidad.

La muestra de información permitió cuantificar las variables o indicadores que forman parte de la investigación y que nos ayudaron a corroborar la hipótesis.

\subsection{Análisis e interpretación de los datos}

Los datos obtenidos se tabularon y procesaron utilizando la hoja de cálculo Excel y la herramienta Visio; posteriormente se diseñó un mo- 
delo de producción modular y se simuló utilizando el lenguaje de programación Awesim. Posteriormente se interpretaron los resultados y se contrastaron con aquellos obtenidos en la situación inicial (método tradicional).

Finalmente, se elaboran conclusiones y recomendaciones en torno a los resultados obtenidos y a los beneficios conseguidos con el sistema de producción modular.

\subsection{Variables utilizadas en la simulación}

- Variables independientes:

- Tiempo estándar de una actividad i-esima (tstdi).

- Tiempo de espera en la estación i-esima (tei).

- Tiempo de preparación o calibración de máquina (tci).

- Tamaño del lote de producción (Qp).

- Tamaño del lote de transferencia (Qt).

- Capacidad presente de producción (Cp).

- Demanda para el periodo "t" (Dt).

- Eficiencia del personal (Ep).

- Polivalencia de los trabajadores.

- Variables dependientes (indicadores)

- Tiempo total de procesamiento (Tp) de un lote.

- Tiempo de reposición de un lote (Tr) de producción.

- Nivel de calidad (N.C).

- Nivel de servicio (N.S).

- Nivel de inventario en proceso (WIP).

- Productividad del recurso humano.

- Utilización de máquinas (Um).

- Productividad global (Pg).

\section{DESCRIPCIÓN DEL PROCESO DE PRODUCCIÓN ACTUAL}

La demanda mensual de la compañía asciende a 20.944 prendas por mes, de las cuales el $80 \%$ se vende en el mercado nacional y el resto se exporta. La proporción de cantidades de prendas T-shirts y S-shirts es de dos a uno.

La línea de producto para estudiar es la línea T-shirt (polos de vestir de manga corta), dicha línea está conformada por diversos modelos 
y colores, los cuales se pueden estandarizar con el modelo "Gino", que se exhibe en el cuadro 1 y cuyas medidas se detallan en tabla adjunta.

La línea de T-shirts utiliza como insumos principales el tejido de algodón, el tejido de punto Rib, el tejido plano (cinta), el hilo, la etiqueta, la bolsa, entre otros.

La empresa tiene 40 trabajadores en planta, los cuales trabajan de lunes a viernes durante un turno por día de 8,5 horas efectivas. Los trabajadores reciben pocas horas de adiestramiento; no se practica el trabajo en equipo; el número de empleados es de 18 personas.

En cuanto a la administración de la producción, se viene aplicando el enfoque de empujar la producción (push production). El lote promedio de producción es de 200 prendas en el corte; dicho orden puede combinar varios pedidos y una diversidad de modelos y tallas. Para el proceso de costura, el habilitado prepara paquetes de partes que pueden contener en promedio 40 prendas.

El sistema actual de producción corresponde al sistema de "bulto progresivo", que da una sola tarea a los operarios y nunca se espera que ellos tomen decisiones. El gerente de producción, el jefe de planta y los supervisores se encargan de todas las actividades relacionadas con el balanceo de la línea, siendo la meta, esencialmente, procurar que cada máquina y cada persona tenga trabajo.

\section{Cuadro 1}

Dimensiones del polo por tallas

\begin{tabular}{lccccc}
\hline \multicolumn{1}{c}{ Descripción } & Letra & S & M & X & XL \\
\hline Ancho delantero & A & 51 & 54 & 57 & 60 \\
Largo delantero & B & 70 & 72 & 74 & 76 \\
Largo de manga & C & 22 & 22 & 24 & 24 \\
Hombro & D & 17 & 18 & 19 & 20 \\
Sisa & E & 25 & 25 & 27 & 27 \\
Boca de manga & F & 18 & 18 & 20 & 20 \\
Abertura de cuello & G & 18 & 19 & 21 & 21 \\
Manga inferior & H & 18 & 18 & 20 & 20 \\
Abertura de pretina & I & 43 & 46 & 49 & 52 \\
Profundidad de cuello & J & 6 & 6 & 7 & 7 \\
Ancho Rib cuello & $\mathrm{K}$ & 2.5 & 2.5 & 2.5 & 2.5 \\
Basta de manga & L & 3 & 3 & 3 & 3 \\
\hline
\end{tabular}

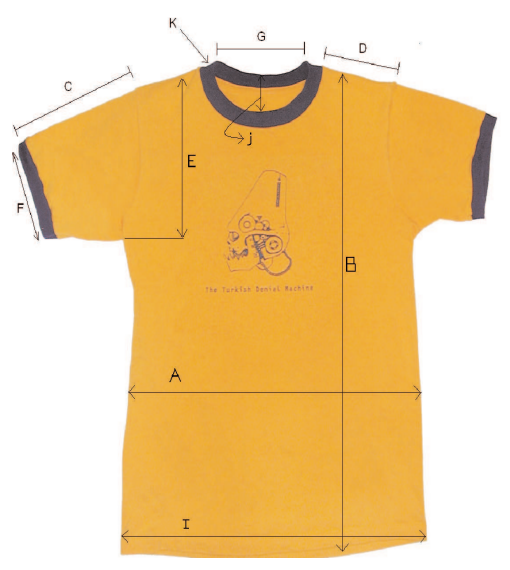


El diagrama de operaciones del producto estándar mostrado se ubica en el anexo 1.

La disposición actual de la planta se muestra en el anexo 2.

\subsection{Diseño modular}

Tomando como referencia la secuencia mostrada en el diagrama de operaciones y la disposición actual, se procede al diseño de la nueva disposición, que se muestra en el anexo 3.

Los cálculos técnicos para el diseño modular se muestran a continuación:

Teniendo una demanda equivalente de 20.944 prendas/mes, necesitamos aproximar el tiempo de ciclo del sistema (Tcy).

$$
\begin{aligned}
& \text { Tcy }=\frac{\text { tiempo efectivo disponible }}{\text { demanda }} \\
& T c y=\frac{22 \frac{\text { días }}{\text { mes }} \times 1 \frac{\text { turno }}{\text { día }} \times 8.5 \frac{\text { horas }}{\text { turno }} \times 0.83\left(^{*}\right) \times 0.935\left(^{* *}\right) \times 60}{20.944 \frac{T-\text { shirt }}{T-\text { shirt }}}=0.42 \frac{\text { min. }}{T}
\end{aligned}
$$

$\left({ }^{*}\right)$ La eficiencia promedio del personal se estima en $83 \%$.

$\left.{ }^{(* *}\right)$ La merma por productos defectuosos se estima en $6,5 \%$.

Los tiempos están en minutos hombre, y los códigos utilizados para las estaciones son:

- Mesa de tendido, tizado y corte: M1

- Mesa de habilitado: MH

- Máquinas remalladoras: R

- Máquinas de costura recta: M

- Máquina recubridora: RC

- Tablero para limpieza de hilos: L

- Planchadora: PL

- Mesa de embolsado y embalado: MA 
Cuadro 2

Relación de actividades, tiempos estándares y máquinas requeridas

\begin{tabular}{|c|c|c|c|c|}
\hline $\begin{array}{c}\mathbf{N}^{\circ} \\
\text { operación }\end{array}$ & $\begin{array}{c}\mathrm{N}^{\circ} \\
\text { Op-Insp. }\end{array}$ & Descripción & $\begin{array}{l}\text { Tiempo } \\
\text { (min-h) }\end{array}$ & $\begin{array}{l}\text { Estación } \\
\text { Máquina }\end{array}$ \\
\hline 1 & & Tendido de tejido de punto & 1.2 & M1 \\
\hline 2 & & Tizas sobre tendido & 0.19 & M1 \\
\hline \multirow[t]{2}{*}{3} & & Cortar tejido punto & 0.45 & M1 \\
\hline & $1^{\prime}$ & Habilitar piezas cortadas & 0.63 & $\mathrm{MH}$ \\
\hline 4 & & Tendido de tejido plano & 0.02 & M1 \\
\hline 5 & & Tizar tejido plano & 0.01 & M1 \\
\hline \multirow[t]{2}{*}{6} & & Cortar tejido plano & 0.02 & M1 \\
\hline & $2^{\prime}$ & Habilitar tejido plano & 0.01 & MA \\
\hline 7 & & Coser y pespuntar trencilla derecho hombro & 0.3 & $\mathrm{R}$ \\
\hline 8 & & Tendido de tejido Rib & 0.1 & M1 \\
\hline 9 & & Tizar sobre tendido & 0.03 & M1 \\
\hline \multirow[t]{2}{*}{10} & & Cortar tejido Rib & 0.05 & M1 \\
\hline & $3^{\prime}$ & Habilitar tejido Rib & 0.06 & $\mathrm{MH}$ \\
\hline 11 & & Pegar cuello a cuerpo & 0.34 & $\mathrm{RC}$ \\
\hline 12 & & Coser tapete en cuello & 0.55 & $\mathrm{R}$ \\
\hline 13 & & Atracar tapete, pespuntar y pegar etiqueta & 0.7 & M \\
\hline 14 & & Coser y remallar trencilla hombro izquierdo & 0.3 & $\mathrm{R}$ \\
\hline 15 & & Bastillar mangas & 0.3 & $\mathrm{R}$ \\
\hline 16 & & Pespuntar mangas & 0.54 & M \\
\hline 17 & & Pegar mangas a cuerpo & 0.67 & $\mathrm{R}$ \\
\hline 18 & & Cerrar cuerpo & 0.64 & $\mathrm{R}$ \\
\hline 19 & & Bastillar orilla del cuerpo & 0.57 & $\mathrm{R}$ \\
\hline \multirow[t]{3}{*}{20} & & Atracar cuello & 0.12 & M \\
\hline & $4^{\prime}$ & Limpiar hilos & 0.75 & L \\
\hline & $5^{\prime}$ & Planchar prenda & 0.63 & $\mathrm{PL}$ \\
\hline 21 & & Doblar & 0.5 & MA \\
\hline 22 & & Embolsar, empaquetar y etiquetar & 0.39 & MA \\
\hline
\end{tabular}

Se han previsto tres módulos: 
- Módulo de corte y habilitado

El dimensionamiento se basa en el criterio de lote económico:

$$
\begin{aligned}
& \text { EOQ corte }=\frac{\sqrt{2(\mathrm{D}) \mathrm{C}}}{\mathrm{H}}=\frac{\sqrt{2 \times(20.944) \times 12 \times(1)}}{8.8}=239 \\
& \text { donde } \mathrm{D}=\text { demanda anual } \\
& \mathrm{C}=\text { costo de lanzar un lote } \\
& \mathrm{H}=\text { costo de inventario: } 0.4 \times(\mathrm{S} / .22)=8.8
\end{aligned}
$$

Aproximamos el número de operarios requeridos de acuerdo con el Tcy

$$
\mathrm{N}^{\circ} \text { op. }(\text { ideal })=\frac{\sum T \text { std }(\text { módulo })}{T \text { cy }(\text { sistema })}=\frac{\sum\left(\text { op. } 1,2,3,1^{\prime}, 4,5,6,2^{\prime}, 8,9,10,3^{\prime}\right)}{0.42 \mathrm{~min} . / \mathrm{T} \text { - shirt }}=6.35=7 \mathrm{op} .
$$

- Módulo de costura

Para aproximar el número de operarios en la sección de costura se tiene:

$$
\mathrm{N}^{\circ} \mathrm{op} .=\frac{\sum \mathrm{Tstd}(\text { costura })}{\mathrm{Tcy}(\text { sistema })}=\frac{5.03 \mathrm{~min}-\mathrm{H} / \mathrm{T}-\text { shirt }}{0.42 \mathrm{~min} . / \mathrm{T}-\text { shirt }}=11.99=12 \mathrm{op} .
$$

\section{Cuadro 3}

Organización del módulo de costura Tcy $\mathbf{0} 0.42 \mathrm{~min} /$ prendas

\begin{tabular}{cccccc}
\hline Operación & Máquina & Tstd & Operario & Tcy (i) (*) & $\begin{array}{c}\text { Utilización } \\
\text { máquina (\%) }\end{array}$ \\
\hline 7 & $\mathrm{R}-1$ & 0.30 & $\mathrm{~A}$ & 0.30 & 71 \\
11 & $\mathrm{R}-1$ & 0.34 & $\mathrm{~B}$ & 0.34 & 81 \\
12 & $\mathrm{R}-2 / \mathrm{R}-3$ & 0.55 & $\mathrm{C} / \mathrm{A}$ ó B & 0.26 & 62 \\
13 & $\mathrm{M}-1 / \mathrm{M}-2$ & 0.70 & $\mathrm{D}-\mathrm{E}$ ó F & 0.35 & 83 \\
14 & $\mathrm{R}-4$ & 0.30 & $\mathrm{E}$ & 0.30 & 71 \\
15 & $\mathrm{R}-5$ & 0.30 & $\mathrm{~F}$ & 0.30 & 71 \\
16 & $\mathrm{M}-3 / \mathrm{M}-4$ & 0.54 & $\mathrm{G} / \mathrm{M}$ & 0.27 & 64 \\
17 & $\mathrm{R}-7 / \mathrm{R}-8$ & 0.67 & $\mathrm{H} / \mathrm{l}$ & 0.34 & 82 \\
18 & $\mathrm{R}-6 / \mathrm{R}-9$ & 0.64 & $\mathrm{~J} / \mathrm{K}$ & 0.32 & 76 \\
19 & $\mathrm{R}-10 / \mathrm{R}-11$ & 0.57 & $\mathrm{~L} / \mathrm{ló} \mathrm{K}$ & 0.29 & 69 \\
20 & $\mathrm{M}-4$ & 0.12 & $\mathrm{M}$ & 0.12 & $29\left(^{* *}\right)$ \\
& & 5.03 & 13 & & \\
\hline
\end{tabular}

(*) Min/prenda.

$\left.{ }^{(* *}\right)$ Se añade a $64 \%$ de utilización de M-4 anotada en la actividad n 6. 
- Módulo de acabado

Las operaciones que se van a desarrollar son: limpieza, acabado, doblado y embolsado.

$$
\mathrm{N}^{\circ} \text { op. }=\frac{\Sigma \text { Tstd }}{0.42}=\frac{2.27}{0.42}=5.4=6
$$

Cuadro 4

Organización de estaciones en el módulo de acabado

\begin{tabular}{ccccc}
\hline No operación $^{\prime}$ & Estación & Tstd & Operario & Tcy \\
\hline 4 & L-1/L-2 & 0.75 & N/O & 0.375 \\
5 & P1/PL-2 & 0.63 & P/Q & 0.315 \\
21 & MA-1 & 0.50 & R/P ó Q & 0.520 \\
22 & MA-2 & 0.39 & S & 0.390 \\
& & & 06 & \\
\hline
\end{tabular}

La utilización promedio del recurso se calcula:

$$
\text { Ef. Op. }=0.337 / 0.42 \times 100=80,24 \%
$$

- Simulación del sistema modular diseñado

El proceso de simulación aplicó el lenguaje de programación de simulación Awesim. El modelamiento utilizó la información descriptiva del proceso productivo y lo acondicionó al sistema modular, tal como se muestra en el layout presentado. El tiempo de simulación ha sido de 22 días, permitiéndonos obtener resultados sobre la producción alcanzada, la utilización de recursos y el nivel de inventarios.

El modelo de simulación ya programado se muestra en el diagrama adjunto. 


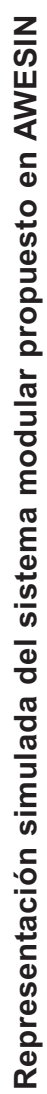

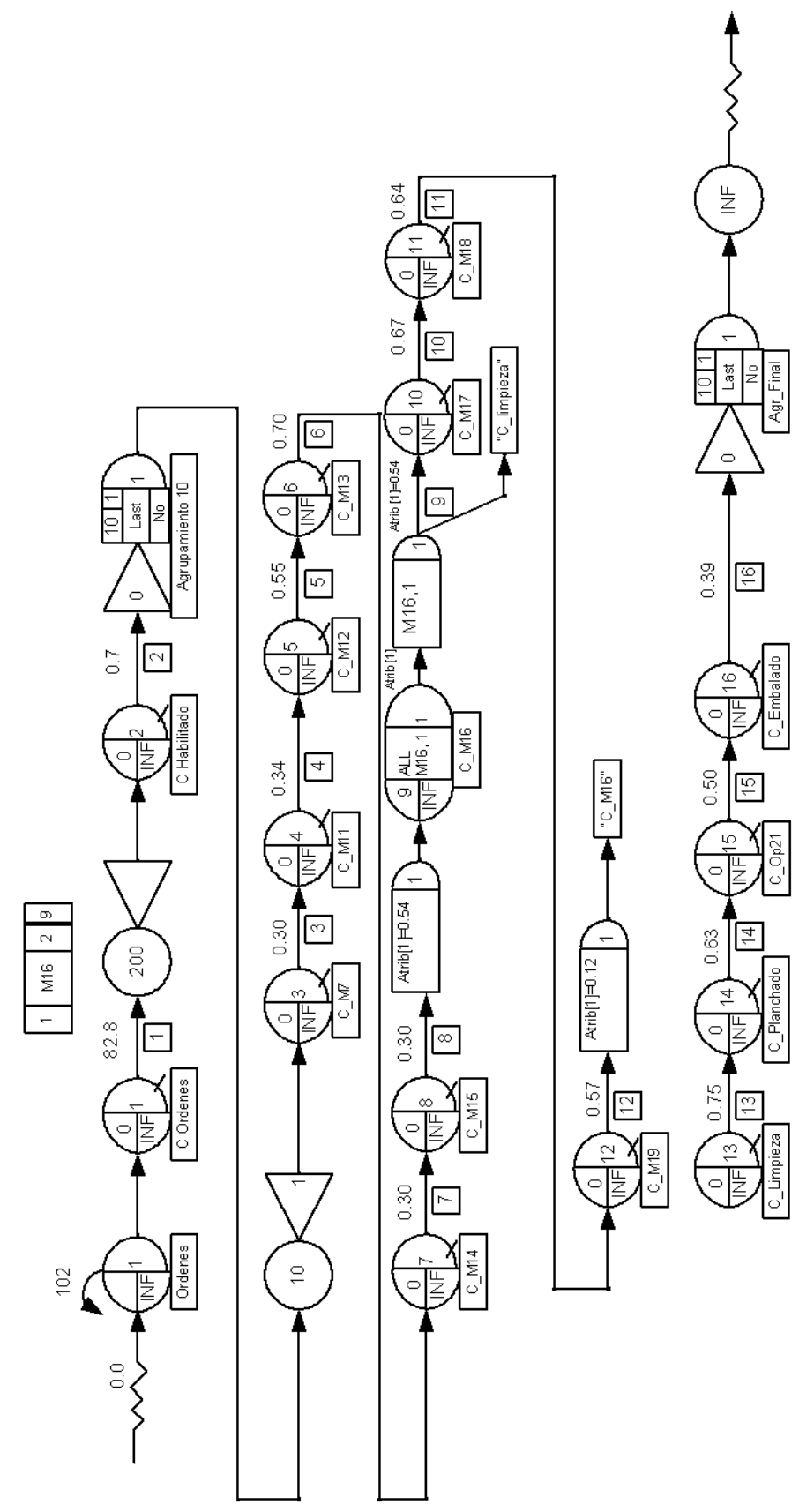




\section{RESULTADOS}

De acuerdo con el reporte mostrado se puede observar que los resultados más importantes son:

\begin{tabular}{lcccc}
\hline \multicolumn{1}{c}{ Indicador } & Unidad & $\begin{array}{c}\text { Sistema } \\
\text { actual (lotes) }\end{array}$ & $\begin{array}{c}\text { Sistema } \\
\text { modular }\end{array}$ & $\begin{array}{c}\% \text { de } \\
\text { mejora }\end{array}$ \\
\hline Productividad de mano de obra & Tshirt / HH & 2.8 & 5.4 & 93 \\
Tiempo de reposición (lead time) & Días & 10.3 & 2 & 81 \\
Nivel de calidad & $\%$ & 6.5 & 3.25 & 50 \\
Nivel de servicio & $\%$ & 67 & 95 & 42 \\
Inventario en Proceso (WIP) & Tshirt /estación & 44 & 3.59 & 92 \\
Número de máquinas de costura & Unidades & 27 & 16 & 41 \\
Utilización de máquinas y equipos & $\%$ & 69 & 93 & 35 \\
Longitud recorrida por prenda & $\mathrm{mt}$ & 40 & 20 & 50 \\
& & & & \\
\hline
\end{tabular}

\section{CONCLUSIONES DE LA INVESTIGACIÓN}

- EI 95\% de las empresas peruanas dedicadas a la confección de prendas de vestir conducen la producción a través del sistema por bultos o por lotes, lo que dadas las exigencias del mercado global resulta ser improductivo e ineficaz.

- Solo el 5\% de empresas peruanas ha modernizado su enfoque sobre la administración de la producción, introduciendo alternativas modernas derivadas de la filosofía "justo a tiempo" (JIT). Esta actitud es muy propia de las grandes empresas de confecciones, como Tejidos San Cristóbal, Topy Top, Nettalco, Cofaco, entre otras. La modernización ha significado cambiar el sistema de producción por bultos (lotes) a sistemas flexibles de flujo continuo, lo que ha requerido la modernización de los equipos y las máquinas, sumado todo esto a un cambio cultural que fomente el trabajo en equipo, la delegación de autoridad y la comunicación.

- La producción modular es un sistema de producción apropiado para aquellos sectores de la producción que requieren flexibilidad, calidad y rápida respuesta a los clientes. Esta forma de producción es parte de los enfoques del lean manufacturing o "producción esbelta".

- El sistema de producción modular en una mediana empresa peruana dedicada a la fabricación de diversos modelos de T-shirts, mejo- 
rará de manera significativa los indicadores importantes en la conducción operativa de la producción: productividad de la mano de obra, tiempo de reposición de un lote de producción, nivel de calidad, inventario en proceso (WIP), número de máquinas y utilización de máquinas y equipos.

- Los ahorros generados por la implementación de un sistema de producción modular, en una mediana empresa de confecciones que factura más de US $\$ 2.000 .000$ por año, representan un $5,7 \%$ de tales ingresos.

- La inversión requerida para la implementación de un sistema de producción modular debidamente implementado, puede recuperarse en el primer semestre de operaciones.

- La implantación de un sistema de producción modular es un rediseño en la programación y conducción operativa del taller, y en ese sentido requerirá como primer paso sensibilizar y comprometer a la alta dirección, y luego transmitir este compromiso a los operarios. Algunos paradigmas que deben ser institucionalizados son: fijar metas de grupo, trabajo en equipo, autobalanceo de la línea de producción por parte de los mismos operarios, transferencia mano a mano en la industria de la confección, sistemas de incentivos grupales, actitud proactiva para la propuesta de mejoras y prevención de errores y empoderamiento con los trabajadores para facilitar la toma de decisión.

- Existe un software en nuestro medio que puede facilitar la simulación del funcionamiento de un sistema de producción modular. Esta primera referencia podrá ser corroborada con la experimentación en módulos pilotos.

- Un módulo puede contener varios submódulos. Este criterio es importante en la fase de experimentación.

- Implementar un sistema de manufactura flexible requiere priorizar las necesidades de cada empresa, ya que estas explotan sus beneficios en aspectos específicos de manera muy particular.

- Tener implementados módulos de producción facilitará la migración del proceso de producción hacia un sistema Kanban; de esta manera, se pasará de empujar (push) la producción, a jalarla (pull). 


\section{RECOMENDACIONES}

- Los sistemas de producción modular pueden combinarse con otras alternativas de conducción operativa, esto dependerá de los nuevos requerimientos del mercado y de la introducción de nuevas tecnologías.

- El éxito de mantener un alto nivel de motivación del personal dependerá de la existencia de un buen liderazgo y un interesante sistema de reconocimientos al trabajo en equipo. Es importante que los equipos de trabajo estén siempre motivados para que se sientan comprometidos con las mejoras y entiendan el beneficio para la empresa y también para ellos.

- La capacitación deberá ser constante, para asegurar que se cuente con trabajadores hábiles en el uso de cualquier tipo de maquinaria, tanto para los trabajadores actuales como para los nuevos.

- La política de incentivos deberá enfocarse en la superación de los tiempos estándares de producción y la calidad de los productos, desde el punto de vista de los resultados grupales y no personales.

- Se recomienda que la implementación de sistemas modulares sea gradual; es muy importante el rol de los módulos pilotos.

- Será beneficioso recurrir a la simulación previa del sistema, para lo cual los empresarios pueden buscar asistencia en universidades o centros tecnológicos de educación superior.

- La gestión logística de la empresa es básica para alcanzar el éxito en la implementación de sistemas modulares. Por un lado, asegura un abastecimiento oportuno de los insumos requeridos, y, por otro, nos brinda información exacta y confiable sobre el comportamiento del mercado.

\section{BIBLIOGRAFÍA}

Domínguez, José Antonio y otros. Dirección de operaciones, aspectos tácticos y operativos en la producción y servicios. Madrid: McGraw-Hill, 1995.

Gaither, Norman y Greg Frazier. Administración de producción y operaciones. México: International Thomson Editores, 2000.

Gutiérrez Garza, Gustavo. Justo a tiempo y calidad total: Principios y aplicaciones. 5. ${ }^{\mathrm{a}}$ edición. Monterrey: Ediciones Castillo S. A. de C. V., 2000. 
Hirano, Hiroyuki. El JIT. Revolución en las fábricas. Madrid: Editorial Tecnologías de Gerencia y Producción S.A., 1992.

Hodson, William K. Manual del ingeniero industrial. Tomo II. 4. ${ }^{\mathrm{a}}$ edición. México: McGraw-Hill, septiembre del 2001.

La Bobina. "Evitando el apocalipsis modular". Julio de 1997, pp. 2832.

—. "Cómo evaluar las distintas alternativas de producción modular-Tercera parte”. Abril de 1997, pp. 37-40.

—. "Cómo evaluar las distintas alternativas de producción modular, segunda parte”. Marzo de 1997, pp. 53-57.

—. "Cómo evaluar las distintas alternativas de producción modular, primera parte”. Febrero de 1997, pp. 37-40.

—. "Programa de capacitación para supervisores. Abril de 1994, pp. 24-26.

_. "Implementación de sistemas de producción modular". Julio de 1993, pp. 44-45.

Nahmias, Steven. Administración de la producción y operaciones. México: CECSA, 1999.

Vollman, Thomas E. y otros. Sistemas de planificación y control de la fabricación. Bogotá: McGraw-Hill, 1997.

Páginas web:

"6 Sigma" [en línea]. <http://www.lean-6sigma.com>.

"Kaizen" [en línea]. <http://www.kaizen-institute.com>. 


\section{ANEXO 1}

\section{Diagrama de flujo para la producción de T-shirt}

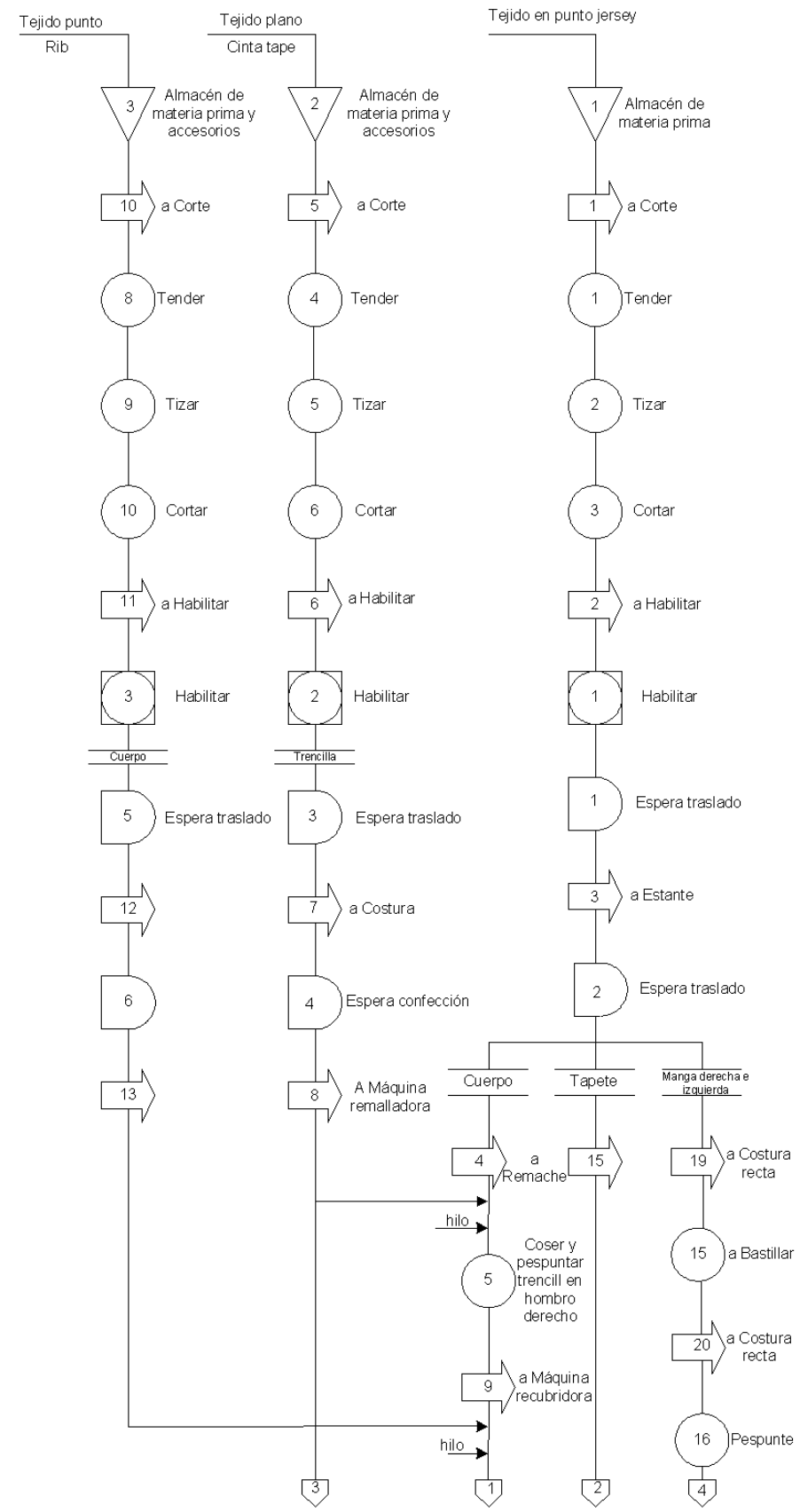


Diseño de un sistema de producción modular en una mediana empresa de confecciones

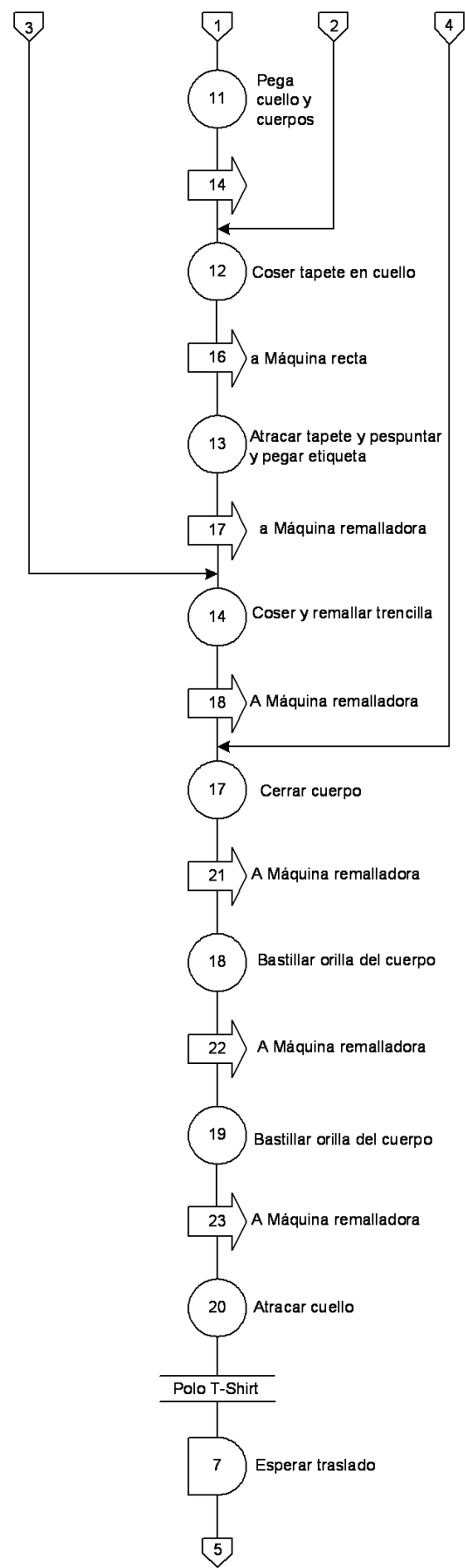




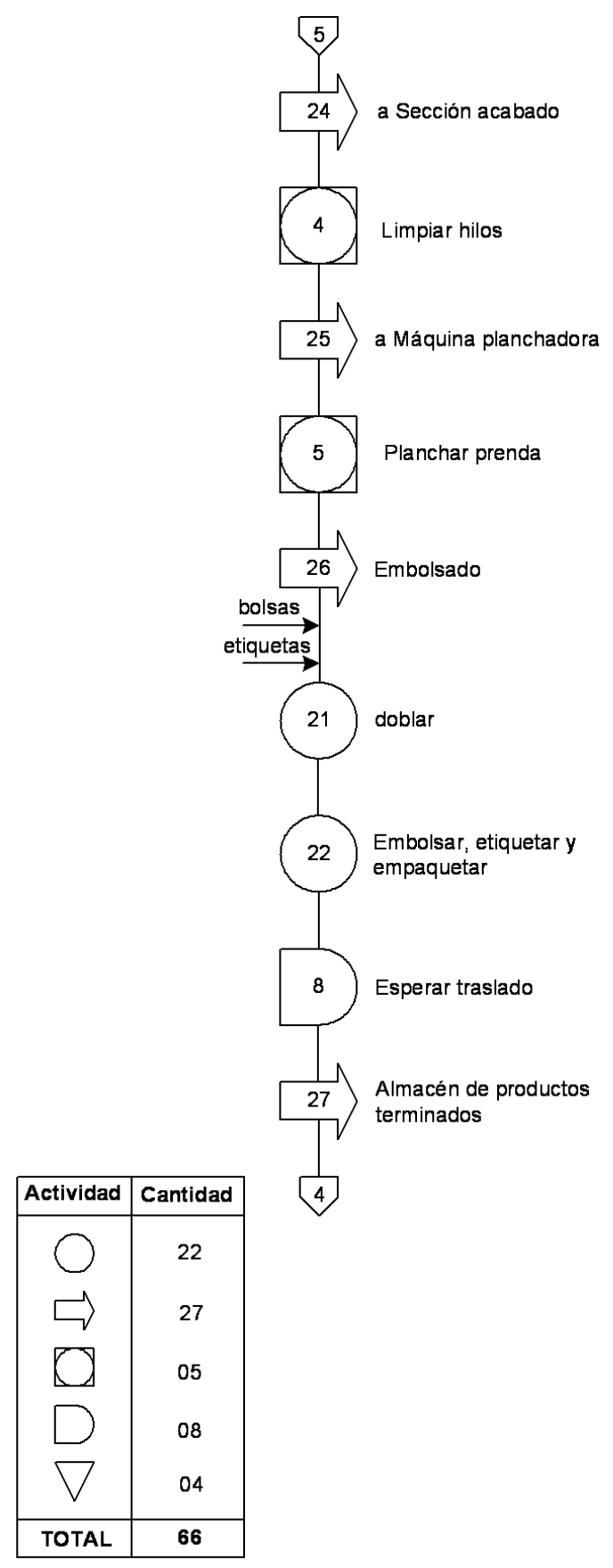




\section{ANEXO 2}

Disposición actual de la planta (Producción por lotes)

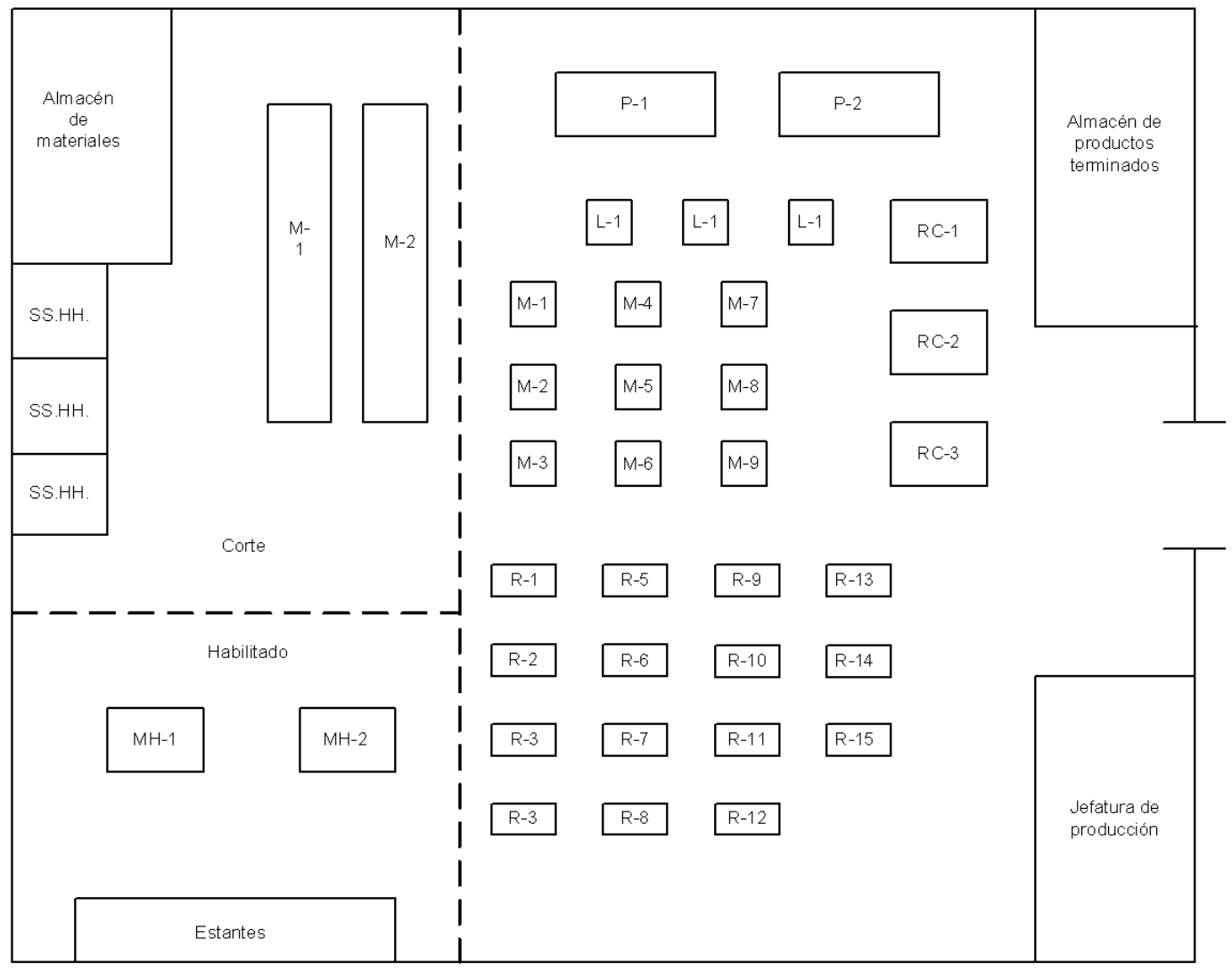


ANEXO 3

\section{Disposición modular}

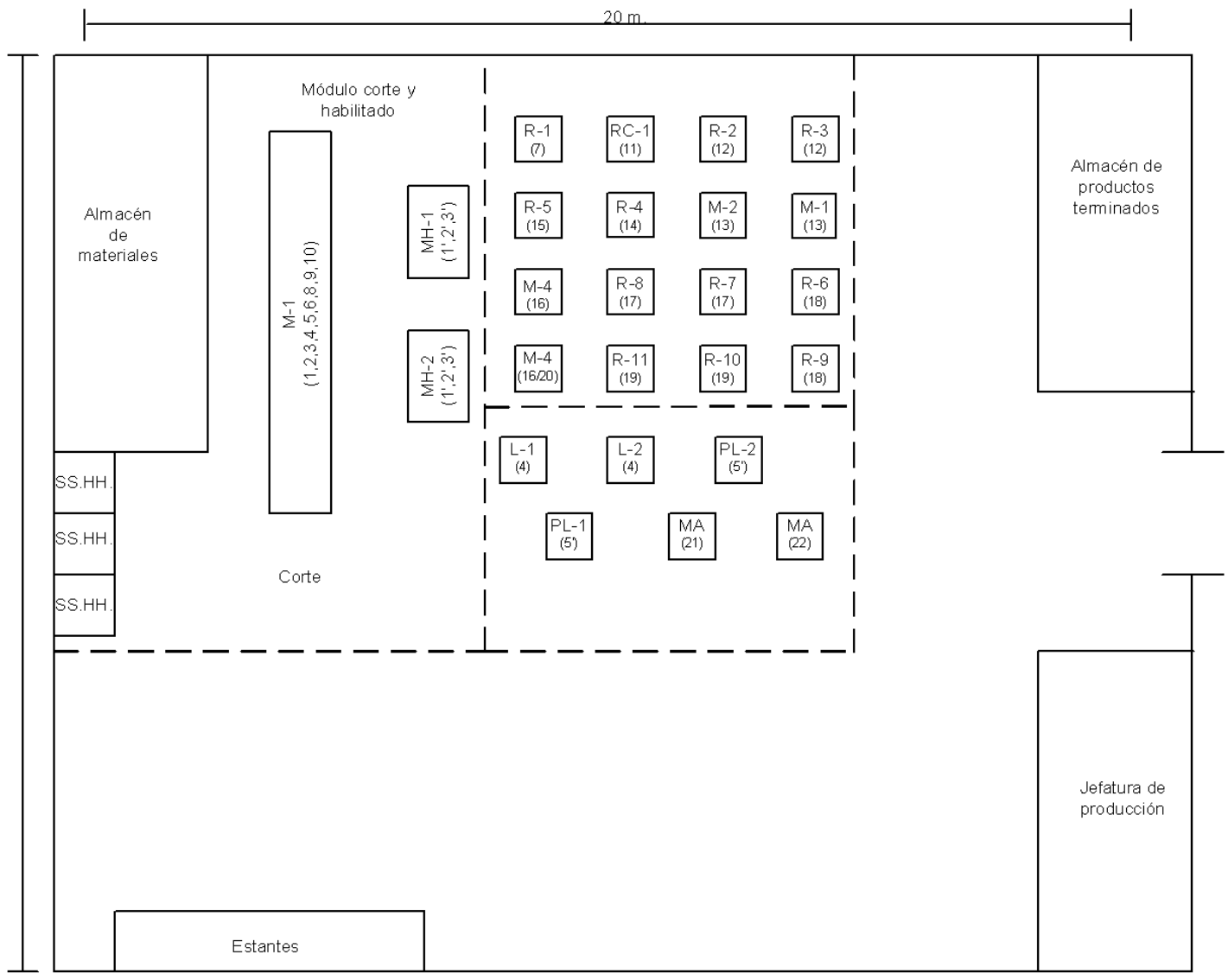

